\title{
Effect of SRD-P401 on the Discomfort of Neck and/or Shoulder Stiffness
}

\author{
Akira Iizuka1* ${ }^{*}$ Maasa Sonoda², Ruka Goto², Tokunori Matsui ${ }^{3}$, Chiaki Kudoh ${ }^{4}$, Katsumi Kido5, \\ Yasuhiro Komatsu ${ }^{3}$
}

${ }^{1}$ Center for Supporting Pharmaceutical Education, Daiich University of Pharmacy, Fukuoka, Japan

${ }^{2}$ Clinical Pharmacy Education Center, Japan College of Pharmaceutical Science, Saitama, Japan

${ }^{3}$ Sun R and D Institute for Natural Medicines Co., Inc., Tokyo, Japan

${ }^{4} \mathrm{KUDOH}$ CHIAKI Clinic for Neurosurgery \& Neurology, Tokyo Brain \& Spinal Cord Research Institute, Tokyo, Japan

${ }^{5}$ Department of Pharmacognosy, Daiich University of Pharmacy, Fukuoka, Japan

Email: *a-iizuka@daiichi-cps.ac.jp

How to cite this paper: Iizuka, A., Sonoda, M., Goto, R., Matsui, T., Kudoh, C., Kido, K. and Komatsu, Y. (2017) Effect of SRDP401 on the Discomfort of Neck and/or Shoulder Stiffness. American Journal of Plant Sciences, 8, 1065-1076.

https://doi.org/10.4236/ajps.2017.85070

Received: March 18, 2017

Accepted: April 23, 2017

Published: April 26, 2017

Copyright $\odot 2017$ by authors and Scientific Research Publishing Inc. This work is licensed under the Creative Commons Attribution International License (CC BY 4.0).

http://creativecommons.org/licenses/by/4.0/

\begin{abstract}
SRD-P401 is a newly developed healthy food supplement which has an anti-inflammatory and analgesic effect. In this report, the effects of SRD-P401 on neck and shoulder stiffness were assessed by an inquiry method in the public field. The stiffness symptoms of all participants were improved after taking SRD-P401. The symptoms of the participants who took $3 \mathrm{~g} / \mathrm{day}$ and/or $9 \mathrm{~g} /$ day of SRD-P401 improved in a week after taking it. Unpleasant adverse effects in stomach and alimentally organs were not found during the test period. SRD-P401 showed the anti-nociceptive effect on the acetic-acid-induces abdominal writhing in rats. The potency was equivalent to aspirin. Moreover, to investigate the ability of the anti-inflammatory effects of SRD-P401, we exerted by inhibiting the Cyclooxygenase (COX) enzyme activity. SRD-P401 and its constituents inhibit the COX activity. The results of this field test indicated SRD-P401 would be a beneficial and useful healthy food supplement for a person with stiffness in neck and/or shoulder.
\end{abstract}

\section{Keywords}

Supplement, Anti-Inflammatory, Anti-Nociceptive, SRD-P401

\section{Introduction}

More than half of the human population in a civilized city has a feeling of abnormality in their health conditions due to various kinds of stress [1]. These stresses influence the physical as well as mental health condition, and would be crucial risk factors for emotional problems such as depression and anxiety. Perceived stress is also known as a risk factor for various diseases such as cardi- 
ovascular disease [2], diabetes [3], asthma [4] and rheumatoid arthritis [5] by stress induced immune suppression and autonomic nervous unbalance. Sustained activation of physiological stress may potentially lead to a reduction in the capacity to respond to new stressors.

In many resent offices, computers are widely used and most of the office workers are performing their office work on the monitor screen of a computer. Therefore, their eyes have been particularly exposed to UV beams and/or micro waves from the monitor display. Office workers are engaging in repetitive monotonous work and have to keep their poor posture in order to continue their work [6]. So, they used to complain about stiffness in neck and shoulder, so called cervix-shoulder arm symptoms, from excessive stress on the optic nerve. The developmental mechanism of those symptoms is not completely understood, but it is said that the blood circulation in the neck and the shoulder could be deficient [7]. As a result of the peripheral blood circulation deficiency, the condition of the neck muscle and the shoulder would be extremely tense. The lactic acid level in the muscle of the neck and the shoulder would be high because of metabolic dysfunction in the tissues with the blood circulation defect.

When people who live in such stressful occupations, would be in "not-illness and/or not-health" conditions, Chinese medicine says this situation is "Mibyou" (in Japanese). If "Mibyou situation (not-illness and/or not-health)" continues for a long time, it might be able to cause an opportunity to induce various kinds of diseases [8]. Therefore, it is important for them to improve their health conditions and try to receive physical treatments such as acupuncture and moxibustion, massage, shiatsu (finger press), chiropractic care, etc. in order to recover their disorders [9]. NSAIDs are mainly used for inflammatory diseases such as muscle relaxants, tranquilizers, rehabilitation, and a plaster patch for orthopedics [10] [11]. It is a well-known fact that the medication of NSAIDs induces quite often adverse reactions in stomach and other alimental organs dysfunctions, such as peptic ulcers, gastritis, etc. Normally, most of the patients have to take such drugs for long periods of time but they could not accept for the long time treatment [12]. The authors obtained an opportunity to examine an effect of a new health food supplement SRD-P401 (a product by Sun R \& D Institute for Natural Medicines Co., Inc. in Tokyo) with analgesics, and anti-inflammatory effects [13]. We conducted a test on the clinical effects of SRD-P401 on stiffness in neck and shoulder by the inquiry method. The information on the SRD-P401 did not match the pharmacological or toxicological information. Therefore, we carried out the acute toxicity and anti-nociceptive effects in mice, and the inhibition activity of cyclooxygenase (COX) in vitro.

\section{Materials and Methods}

\subsection{Materials}

SRD-P401 prepared as a mixture of 12 herbs which are Citrus unshiu peel, Cinnamon bark, Red ginseng, Pine bark, White willow bark, Jujube, Citrus junos seed, Licorice root, Gardenia fruit, Ginger, adlay and Antrodia camphorata. 


\subsection{Clinical Experiment of SRD-P401 on the Stiffness of Shoulder and Neck}

This clinical test was approved by KUDOH CHIAKI Clinic for Neurosurgery \& Neurology, Tokyo Brain \& Spinal cord Research Institute, Tokyo, Japan.

\section{The Background of the Participants}

The total number of the participants was 30 people in the two groups $A$ and $B$, and both $\mathrm{A}$ and $\mathrm{B}$ groups had 15 participants. 14 participators out of $30 \mathrm{did}$ not return their questionnaires. Therefore, 16 participants were assessable. 8 participants in the each group were analyzed for the efficacy. There were three men and four women in the Group A, and two men and six women in Group B. Their age was between 37 years and 68 years old in the group A, and in the group B their age was between 16 years and 65 years old.

5 participants complained of pain in their loins, shoulder and neck and the other participants had pain in their shoulder and neck because of the stiffness. This study would clarify that loins, shoulder and neck are common sites of pain occurred in the body. In group A, the two participants were diagnosed with sciatica, and one had chronic lumbago and disk herniation. Other three participants did not name their disease. One was spondylolisthesis and another one had a traffic accident. Most of the participants complained about stiffness every day, and their pain might be very difficult phenomenon (Table 1).

1) Enrolment of applicants

The organizer invited applicants with the neck and shoulder stiffness from the Table 1. Background of the participant.

\begin{tabular}{|c|c|c|c|c|c|}
\hline \multicolumn{2}{|c|}{ Group } & \multirow{2}{*}{$\begin{array}{l}\text { Gender } \\
\text { Female }\end{array}$} & \multirow{2}{*}{$\begin{array}{l}\text { Age } \\
45 \mathrm{y}\end{array}$} & \multirow{2}{*}{$\begin{array}{c}\text { Name of disease } \\
\text { Sciatica }\end{array}$} & \multirow{2}{*}{$\begin{array}{c}\text { Part of stiffness } \\
\text { Neck, shoulder, loin }\end{array}$} \\
\hline A & 1 & & & & \\
\hline & 2 & Male & $48 \mathrm{y}$ & Sciatica & Shoulder, loin \\
\hline & 3 & Male & $62 \mathrm{y}$ & Constitution & Neck, shoulder \\
\hline & 4 & Male & $16 \mathrm{y}$ & Not reported & Shoulder \\
\hline & 5 & no answer & $67 y$ & Not reported & Neck \\
\hline & 6 & Female & $35 \mathrm{y}$ & Chlonic lunbago & Neck, shoulder, loin \\
\hline & 7 & Female & $26 y$ & Not reported & Neck, shoulder \\
\hline & 8 & Female & $68 \mathrm{y}$ & Spondylolisthesis & Neck, shoulder, loin \\
\hline \multirow[t]{8}{*}{ B } & 1 & Female & $54 \mathrm{y}$ & Not reported & Neck, shoulder \\
\hline & 2 & Female & & Traffic acident & Neck, shoulder \\
\hline & 3 & Female & $57 \mathrm{y}$ & Not reported & Neck, shoulder \\
\hline & 4 & Female & $40 \mathrm{y}$ & Constitution & Neck \\
\hline & 5 & Male & $37 \mathrm{y}$ & Other & Neck, shoulder \\
\hline & 6 & Female & $64 \mathrm{y}$ & Constitution & Neck, shoulder \\
\hline & 7 & Male & $65 y$ & Acute bad back & Neck, shoulder \\
\hline & 8 & Female & $41 \mathrm{y}$ & Constitution & Neck, shoulder, loin \\
\hline
\end{tabular}


general public.

In this study, the enrolled subjects had no limitation in terms of gender and age and they had to have a feeling of "stiffness" in "neck" and "shoulder" due to continuance of the muscular tension due to physical work. Applicants offered a self-declaration form to state their "stiffness condition in neck and/or shoulder.

2) Intake volume and period

All participants could choose to be in either group A or B.

SRD-P401 was administered at doses of $1 \mathrm{~g}$ (Group A) and $3 \mathrm{~g}$ (Group B) for three times a day. All participants took the tablets for one week regardless of a medical history of the disease and they could take the tablets when they wanted it any time because the organizer did not fix the particular taking time, because it is a food and not a medicine.

3) The evaluation of the SRD-P401 effect

The evaluation of effectiveness of SRD-P401 was described by self-assessment in a questionnaire survey of the study report.

Their self-assessment expressed as "VAS score" that described the degree of feeling about "stiffness" of the "neck" and "shoulder" after the intake.

They marked (V) their degree of their "stiffness" on the VAS scale once a day.

4) The investigation of adverse events

When diarrhea, and/or some unfavorable effects including alimentary canal symptoms including constipation were found through the intake period, they had to describe the event(s) in a questionnaire.

5) Cancellation of the intake

i) When the participants have anxiety about taking the tablets because of a feeling of physical or mental incongruity in their body.

ii) When the participants have not found any effectiveness of SRD-P401 on the "neck" "shoulder stiffness and they do not want to take it anymore.

iii) When the participants want to change to other medication because of no expectation to continue to take it.

iv) When their doctors deicide to give other rescue treatment because of its ineffectiveness.

v) When participants want to cancel stop the trial.

6) Inhibition of combination therapy with other drugs

The use of steroidal and non-steroidal anti-inflammatory drugs was prohibited during the study. These drugs would influence the assessment of SRD-P401 effect on "stiffness symptoms" of the "neck" and "shoulder".

\subsection{Toxicity and Anti-Nociceptive Effect in Mice}

1) Animals

Four-week old male ICR mice (JAPAN SLC) were used. The animals were housed where the temperature and relative humidity were kept at $23^{\circ} \mathrm{C} \pm 2{ }^{\circ} \mathrm{C}$ and $55 \% \pm 10 \%$ with lights on between AM 7:00 and PM 7:00. The animals were allowed free access to food and drinking water. Research protocols were approved by the Animal Care and Welfare Committee of Nihon Pharmaceutical 
University and were performed according to the animal care guidelines of the National Institutes of Health.

2) Acute toxicity study

The toxic effects of the extracts of SRD-P401 were evaluated before further experimentation. The SRD-P401 extract were analyzed for acute toxicity profile with reference to any behavioral changes and mortality in Wister rats. The protocols were followed according to internationally accepted OECD-423 guidelines. The animals were randomly divided in to six groups with ten animals in each. A dose of $8000 \mathrm{mg} / \mathrm{Kg}$ were administered orally. All animals were fasted 18 hours prior to treatment but had free access to water. After administration of the test drugs, sign of toxicity and mortality was observed with special attention once an hour for the first four hours and daily four times thereafter for a period of fourteen days. Any symptoms of ill health or mortality were recorded.

3) Effect on the acetic-acid-induced abdominal writhing

Animals were administered orally with SRD-P401 extraction (100 and 1000 $\mathrm{mg} / \mathrm{kg})$ or aspirin $(100 \mathrm{mg} / \mathrm{kg})$ before intraperitoneal administration with $6 \%$ acetic acid solution. The number of writhings was recorded for $10 \mathrm{~min}$ after injection of acetic acid and expressed as a percentage relative to the number of writhings in vehicle controls. $\mathrm{P}<0.05$ compared with the control.

\subsection{Inhibition of the Cyclooxygenase Activity}

The COX activity was measured by COX Activity Assay Kit (Cayman Chemical, MI) at a dose of $100 \mathrm{mg} / \mathrm{mL}$.

\subsection{Statistical Analysis}

Data were evaluated by analysis of variance (ANOVA), $\mathrm{p}<0.05$ was considered significant.

\section{Results}

\subsection{Evaluation of Effectiveness}

The evaluation of SRD-P401 efficacy is shown in Figure 1.

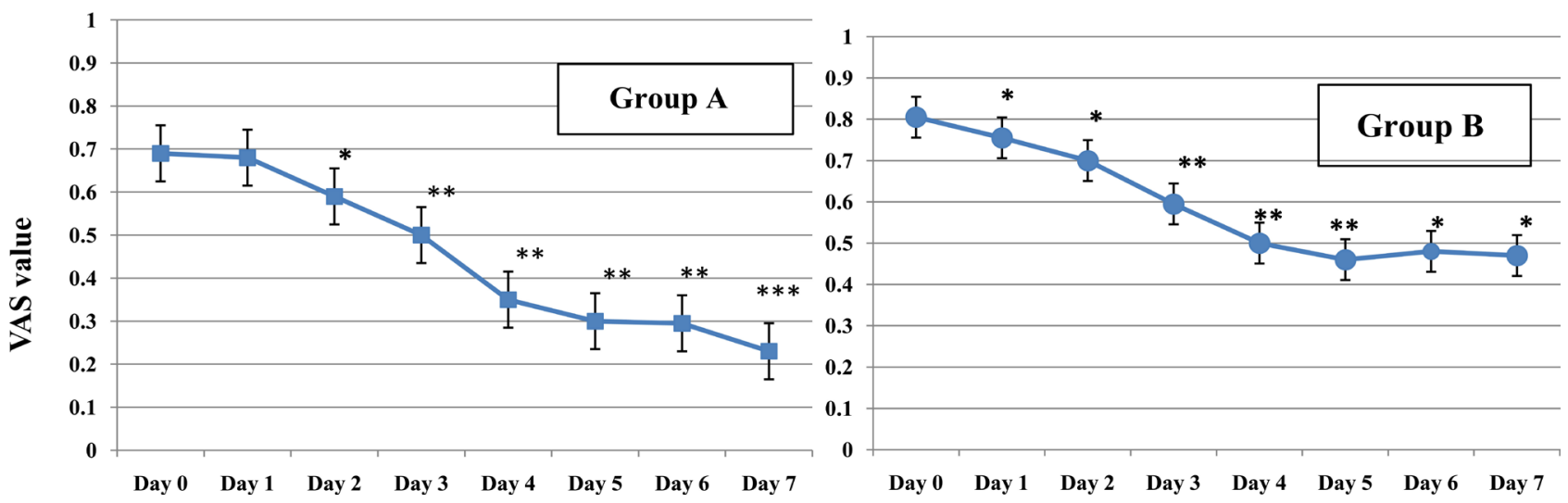

Figure 1. The evaluation of SRD-P401 efficacy. The VAS score of "neck stiffness and the shoulder discomfort" in the group A and B. Each value represents the mean \pm SE. 
The score of "neck stiffness and the shoulder discomfort" in the group A began to significantly decrease after SRD-P401 intake from day 2, and the original neck stiffness and shoulder discomfort in the group decreased in $50 \%$ by day 4 . The result indicated that the neck stiffness and the shoulder discomfort would be cured by the end of the study. On the other hand, the score in the group B was significantly decreasing after SRD-P401 intake from day 1, and the original neck stiffness and shoulder discomfort in the group decreased by $40 \%$ by day 5 .

One participant complained of stomachache and left from the study. This was a dropout case. Two participants quit to take the tablets because of not finding any efficacy of SRD-P401 (SRD-P401) on the "neck stiffness and shoulder discomfort". B-7 and B-6 participant stopped to take the tablets on day 6 and day 5, respectively. The other participants in the group B completed this study. They showed apparent decrease in the score of "the neck stiffness and the shoulder discomfort.

\subsection{Safe Evaluation}

During SRD-P401 intake, there was no finding of severe adverse reactions in alimental organs in the 15 participants who took the SRD-P401 tablets through the study. They did not complain about any alimental organs defects. There were no adverse reactions observed. SRD-P401 was thought to be safe but one participant had constipation, and another one, however, diversely cured soft feces. One participant who complained of stomachache belched after taking SRD-P401 tablets in early study days. However, during through the study time there was no-one who needed to take any stomach digestive medicines.

\subsection{Acute Toxicity}

In the acute toxicity test of the SRD-P401, there was no mortality or any signs of behavioral changes or pharmacotoxicity observed after oral administration of the different doses even to the highest dose administered ( $8 \mathrm{~g} / \mathrm{kg}$ body weight) in rat until the end of the study period.

Anti-nociceptive Effect of SRD-P401 in Writhing Test

The pretreatment of mice with SRD-P401 1000mg/kg caused a significant decrease in number of abdominal constrictions compared to the control (Table 1 \& Table 2). The percentage of inhibition of constriction was calculated as $63.5 \%$.

Table 2. Anti-nociceptive activity of SRD-P401 in acetic acid-induced abdominal constriction test in mice.

\begin{tabular}{|c|c|c|c|c|}
\hline Treatment & Dose & No of writhing & & $\%$ Inhibition \\
\hline Vehicle & $10 \mathrm{~mL} / \mathrm{kg}$ & $11.00 \pm 0.89$ & & \\
\hline SRD-P401 & $1000 \mathrm{mg} / \mathrm{kg}$ & $3.83 \pm 0.48$ & $(\mathrm{p}<0.05)$ & 65.2 \\
\hline SRD-P401 & $100 \mathrm{mg} / \mathrm{kg}$ & $5.80 \pm 3.38$ & & 47.3 \\
\hline Aspirin & $100 \mathrm{mg} / \mathrm{kg}$ & $3.41 \pm 2.01$ & $(\mathrm{p}<0.05)$ & 69.0 \\
\hline
\end{tabular}

Each value represents as mean $\pm \mathrm{SE}(\mathrm{N}=5$ - 6) 
Aspirin $(100 \mathrm{mg} / \mathrm{kg})$ showed significantly inhibition of abdominal constriction $(69 \%)$.

\subsection{Inhibition of COX, COX-1 and COX-2 Activity}

We examined the inhibitory activity of COX, COX-1 and COX-2, about SRDP401, 7 kinds of herb powder and 5 kinds of herb extract at concentration of 100 $\mathrm{mg} / \mathrm{mL}$. The SRD-P401 completely inhibited the COX and COX-1 activity. All the samples except the powder of Citrus unchiu peel, Pine bark and Citrus junos seed, and the extract of CInnamon bark, completely inhibited COX activity. Moreover, the inhibition of COX-1 activity showed all samples except the powder of Pine bark. However, the inhibition of COX-2 activity showed the powder of Pine bark and Citrus junos seed and extract of Gardenia fruit, Red ginseng and White willow bark.

\section{Discussion}

It is imperative to conduct the toxicity study for safety and efficacy of the herbal product. From the acute toxicity study conducted for aqueous extract of SRDP401, no mortality or any behavioral changes were observed in tested animals. The result showed that administration of dose level up to $8000 \mathrm{mg} / \mathrm{Kg}$ body weight was safe.

Cervix-shoulder arm symptoms, neck and shoulder stiffness, are one of the difficult symptoms to cure in the well developed countries.

Their "stiffness" was due to the mental and physical stress including a change of the adaptation ability for the stresses by the decreased biological function of manual labor, muscle fatigue and the deficiency of blood circulation. These symptoms chronically happen to the people who have been receiving chronic stress in the working place [14]. Generally, most medical doctors give patients the neck and shoulder stiffness NSAIDs medication [15]. Many NSAIDS are clinically used for inflammatory diseases. These treatments, however, are not always effective on the stiffness phenomena. It is quite difficult to cure the stiffness, cervix-shoulder arm symptoms right now. The authors have been looking for a new agent including a healthy food supplement for the treatment instead of NSAIDs and/or muscle relaxants. Actually, we really need something to prescribe for patients which do not have any adverse reactions for the long time use.

A new healthy food supplement, SRD-P401 with analgesic and anti-inflammatory effect would be expected as a useful and beneficial agent for a medication to such patients in terms of the safety for a long term treatment. The authors conducted a clinical trial of the supplement in order to assess its efficacy on cervix-shoulder arm symptoms in the field by the inquiry test. In this study, 30 participants were recruited from the general public but unfortunately around the half of the entrants did not return the inquiry sheet to us. Therefore, SRD-P401 efficacy has been analyzed in 16 participants. The mean original pain score of the group B was 0.81 and rather higher levels than the score of the group A 0.64 and this meant the pain in the group B was more severe than that of group $A$ 
participants. However, it was thought that their physiological and pathological phenomena were considered to be no difference between group A and group B. Only their pain severity was not the same among them. Why the difference has been occurred? Because the participants in the group A who did not complained very much their severity of neck stiffness and the shoulder discomfort, then they probably chose the low dose intake. But the participants in group B chose the high dose intake because of feeling their neck stiffness, and the shoulder discomfort to be very severe. The decrease in "the score of neck stiffness and the shoulder discomfort" in both groups started early after taking SRD-P401. Significant decrease in the pain score was shown from day 2 after administration in the group B and the participants in group A exhibited the pain score decrease on day 1 . Surprisingly, the manifestation of the effect happened immediately after the administration. Reasons why this response quickly occurred were not clear. It could be explained maybe by quick absorption of active substances in SRDP401 from alimental organs.

It could be considered that saponin like compounds in this formulation would contribute to quick absorption of active substances from alimental organs. In Kampo medicines (Japanese herbal medicine) or Chinese Traditional Medicines, active ingredients are absorbed very quickly [16]. Such prompt expression of analgesic effect could be because the health food supplement would contain a lot of effective substances with anti-inflammatory effect. However, the correct reason of those phenomena must be determined by future studies. In our data, SRD-P401 produced a significant anti-nociceptive effect in the writhing test.

The authors, also, have been performing veterinary clinical study about the effect of the health supplement on dogs and cats with painful joint disease. Osteoarthritis is a very common disease in aged dogs like aged humans have and in the cases of aged dogs the canine arthritis are common in osteoarthritis of spine from the characteristic structure of the animal. Effects of SRD-P001, a new type of health food supplement, were investigated on the joint diseases in dogs [17]. On other pain symptoms except for joint diseases in dogs or cats, Katagi M. gave SRD-P401 to dogs after surgical operations on breast cancer in order to control their pain and Toyonaga S. treated dogs after exodontia. They reported SRDP401 controlled the pain very well without any adverse reactions [13]. Since SRD-P401 has been applied to dogs, cats and human for over 5 years, there have been no reported adverse reactions in the digestive organs, so far. This suggested it would be a quite safe heath food supplement.

This supplement contains 12 herbs which are all food materials that are known as western and oriental herbs. They have biological effects such as analgesic, anti-inflammatory and anti-oxidative effect and these effects have been analyzed and reported in the science journals. The effects of the herbal remedies could be expected from the results of the previous published papers. In the constituent herbs of SPD-P401, 4 herbs such as Gardenia fruit, Red ginseng, White willow bark and Jujube, have a potent inhibitory effects of both COX-1 and COX-2. 
Gardenia fruit extract (GfE) inhibited vascular permeability in a rat experimental inflammation induced by acetic acid [18]. GfE caused a dose dependent inhibition manner in an acetic acid induced abdominal writhing in mice [19]. This activity expresses analgesic and anti-inflammatory effect of GfE that also inhibits platelet aggregation by phospholipase 2 inhibition [20]. The secretions of nitric oxide and prostaglandin E2 were inhibited by genipin with concentration dependent manner [21]. From the results, genipin or geniposide in GfE are considered to be active compounds. Therefore, it inhibits arachidonic acid metabolism and inflammation.

Jujube has been employed as an essential orient folk medicine for thousands of years. The extract of jujube was investigated for its anti-inflammatory effect using acute and chronic models of inflammation in rat. Acute inflammation was induced by subplantar administration of carrageenan, and chronic inflammation was induced by interscapular implantation of a sterile cotton pellet. Jujube extract has anti-inflammatory effects on both acute and chronic inflammation [22]. The aqueous extract of the Jujube root bark has potent anti-allergic and anti-inflammatory due to inhibition of the COX-1, COX-2, and 5-Lipoxygenase activity [23].

It has been reported that ginseng has a wide range of pharmacological activities in cardiovascular [24], endocrine [25], immune [26], and central nervous systems [27]. It is especially well established that ginseng ameliorates inflammatory responses in allergic asthma model [28], atherosclerosis [29] and many models [30] [31].

The extract of willow bark as an analgesic agent has been used for patients with arthralgia in Europe even at the present time [32]. From ancient time, this herb had been used by people as a "Pain Killer" plant that contains the salicylic acid related compounds and gave us an opportunity of discovery for the salicylic compounds. European people are still using it as a "Pain Killer" health food supplement and studying on the biological activities [33].

Most of the herbs show anti COX I and COX II inhibitor. The remedy has a dual inhibition of arachidonic acid metabolism in the inflammation area. The mechanism of SRD-P401 action has not been clarified because fundamental research has not been conducted yet.

The further investigational clinical studies would be expected to clarify the efficacy in future.

\section{References}

[1] Lundberg, U. (2005) Stress Hormones in Health and Illness: The Roles of Work and Gender. Psychoneurocrinology, 30, 1017-1021.

[2] Kloet, A.D., Wang, L., Pitra, S., Hiller, H., Smith, J.A., Tan, Y., Nguyen, D., Cahill, K.M., Sumners, C., Stern, J.E. and Krause, E.G. (2017) A Unique “Angiotensin Sensitive” Neuronal Population Coordinates Neuroendocrine, Cardiovascular and Behavioral Responses to Stress. Journal of Neuroscience, 37, 3478-3490.

[3] Harris, M.L., Oldmeadow, C., Hure, A., Luu, J., Loxton, D. and Attia, J. (2017) Stress Increases the Risk of Type 2 Diabetes Onset in Women: A 12-Year Longitudi- 
nal Study Using Causal Modelling. PLoS ONE, 12, e0172126.

https://doi.org/10.1371/journal.pone.0172126

[4] Loerbroks, A., Ding, H., Han, W., Wang, H., Wu, J.P., Yang, L., Angerer, P. and Li, J. (2017) Work Stress, Family Stress and Asthma: A Cross-Sectional Study among Women in China. International Archives of Occupational and Environmental Health, 1-8. https://doi.org/10.1007/s00420-017-1201-7

[5] Brandstetter, S., Riedelbeck, G., Steinmann, M., Ehrenstein, B., Loss, J. and Apfelbacher, C. (2017) Pain, Social Support and Depressive Symptoms in Patients with Rheumatoid Arthritis: Testing the Stress-Buffering Hypothesis. Rheumatology International, 1-6, https://doi.org/10.1007/s00296-017-3651-3

[6] Jay, K., Brandt, M., Hansen, K., Sundstrup, E., Jakobsen, M.D., Schraefel, M., Sjogaard, G., Sci, M. and Andersen, L.L. (2015) Effect of Individually Tailored Biopsychosocial Workplace Interventions on Chronic Musculoskeletal Pain and Stress among Laboratory Technicians: Randomized Controlled Trial. Pain Physician, 18, 459-471.

[7] Matsumoto, H., Takenami, E., Iwasaki-Kurashige, K., Osada, T., Katsumura, T. and Hamaoka, T. (2005) Effects of Blackcurrant Anthocyanin Intake on Peripheral Muscle Circulation during Typing Work in Humans. European Journal of Applied Physiology, 94, 36-45. https://doi.org/10.1007/s00421-004-1279-y

[8] Kimura, T., Tsuda, Y., Uchida, S. and Ebishida, A. (2006) Association of Perceived Stress and Stiff Neck/Shoulder with Health Status: Multiple Regression Models by Gender. Hiroshima Journal of Medical Sciences, 55, 101-107.

[9] Donoyama, N. and Shibasaki, M. (2010) Differences in Practitioners Proficiency Affect the Effectiveness of Massage Therapy on Physical and Psychological States. Journal of Bodywork and Movement Therapies, 14, 239-244.

[10] Williamson, C.M. (1983) Clinical Evaluation of Piroxicam in Acute Musculoskeletal Injuries Seen in General Practice. Current Medical Research and Opinion, 8, 622625. https://doi.org/10.1185/03007998309109808

[11] Quijada-Carrera, J., Valenzuela-Castaño, A., Povedano-Gómez, J., Fernández-Rodriguez, A., Hernánz-Mediano, W., Gutierrez-Rubio, A., de la Iglesia-Salgado, J.L. and García-López, A. (1996) Comparison of Tenoxicam and Bromazepan in the Treatment of Fibromyalgia: A Randomized, Double-Blind, Placebo-Controlled Trial. Pain, 65, 221-225.

[12] Inaba, T., Ishikawa, S., Miyoshi, M. and Kurahara, K. (2013) Present Status of Gastrointestinal Damage Due to Non-Steroidal Anti-Inflammatory Drugs (NSAIDs). Nihon Rhinsho, 71, 1109-1115.

[13] Katagi, M., Yasukawa, A. and Komatsu, Y. (2012) Clinical Application of a Health Food Supplement, SRD-P401, in Dogs and Cats. The Journal of Veterinary Oriental Medicine, 19, 15-20.

[14] Metikaridis, T.D., Hadjipaviou, A., Artemiadis, A., Chrousos, G. and Darviri, C. (2017) Effect of a Stress Management Program on Subjects with Neck Pain: A Pilot Randomized Controlled Trial. Journal of Back and Musculoskeletal Rehabilitation, 30, 23-33. https://doi.org/10.3233/BMR-160709

[15] Allison, G.T., Nagy, B.M. and Hall, T. (2002) A Randomized Clinical Trial of Manual Therapy for Cervico-Brachial Pain Syndrome-A Pilot Study. Manual Therapy, 7, 95-102. https://doi.org/10.1054/math.2002.0453

[16] Chen, Y., Wang, J., Yuan, L., Zhou, L., Jia, X. and Tan, X. (2011) Interaction of the Main Components from the Traditional Chinese Drug Pair Chaihu-Shaoyao Based on Rat Intestinal Absorption. Molecules, 16, 9600-9610.

https://doi.org/10.3390/molecules 16119600 
[17] Yasukawa, A., Sano, J., Uno, M., Ogata, M. and Komatsu, Y. (2012) Clinical Application of a Health Food Supplement, SRD-P001, in Dogs and Cats. The Journal of Veterinary Oriental Medicine, 9, 2-9.

[18] Ni, H.Y., Zhang, Z.H. and Fu, H.Z. (2006) Research and Development of Fructus Gardeniae. China Journal of Chinese Materia Medica, 31, 538-541.

[19] Suzuki, Y., Kondo, K., Ikeda, Y. and Umemura, K. (2001) Antithrombotic Effect of Geniposide and Genipin in the Mouse Thrombosis Model. Planta Medic, 67, 807810. https://doi.org/10.1055/s-2001-18842

[20] Wang, Q.S., Xiang, Y., Cui, Y.L., Lin, K.M. and Zhang, X.F. (2012) Dietary Blue Pigments Derived from Genipin, Attenuate Inflammation by Inhibiting LPS-Induced iNOS and COX-2 Expression via the NF- $\kappa$ B Inactivation. PLOS ONE, 7, e34122. https://doi.org/10.1371/journal.pone.0034122

[21] Koo, H.J., Lim, K.H., Jung, H.J. and Park, E.H. (2006) Anti-Inflammatory Evaluation of Gardenia Extract, Geniposide and Genipin. Journal of Ethnopharmacolog, 103, 496-500.

[22] Yu, L., Jiang, B.P., Luo, D., Shen, X.C., Guo, S., Duan, J.A. and Tang, Y.P. (2012) Bioactive Components in the Fruits of Ziziphus jujube Mill. against the Inflammatory Irritant Action of Eupherboa plants. Phytomedicine, 19, 239-244.

[23] Talmale, S., Bhujade, A. and Patil, M. (2015) Anti-Allergic and Anti-Inflammatory Properties of Zizyphus mauriana Root Bark. Food \& Function, 6, 2975-2983. https://doi.org/10.1039/C5FO00270B

[24] Lee, C.H. and Kim, J.H. (2014) A Review on the Medical Potentials of Ginseng and Ginsenosides on Cardiovascular Diseases. Journal of Ginseng Research, 38, 161166.

[25] Lee, H.W., Choi, J., Lee, Y., Ki, K.J. and Lee, M.S. (2016) Ginseng for Managing Menopausal Woman's Health. A Systematic Review of Double-Blind, Randomized, Placebo-Controlled Trials. Medicine, 95, e4914. https://doi.org/10.1097/MD.0000000000004914

[26] Kang, S. and Min, H. (2012) Ginseng, the "Immunity Boost": The Effects of Panax ginseng on Immune System. Journal of Ginseng Research, 36, 354-368. https://doi.org/10.5142/jgr.2012.36.4.354

[27] Rokot, N.T., Kairupan, T.S., Cheng, K.C., Runtuwene, J., Kapantow, N.H., Amitan, M., Morinaga, A., Amitani, H., Asakawa, A. and Inui, A. (2016) A Role of Ginseng and Its Constituents in the Treatment of Central Nervous System Disorders. Evidence-Based Complementary and Alternative Medicine, 2016, Article ID: 2614742. https://doi.org/10.1155/2016/2614742

[28] Kim, D.Y. and Yang, W.M. (2011) Panax Ginseng Ameliorates Airway Inflammation in an Ovalbumin-Sensitized Mouse Allergic Asthma Model. Journal of Ethnopharmacology, 136, 230-235.

[29] Lee, J., Cho, Y. and Kim, W.K. (2014) Anti-Inflammation Effect of Exercise and Korean Red Ginseng in Aging Model Rats with Diet-Induced Atherosclerosis. Nutrition Research and Practice, 8, 284-291. https://doi.org/10.4162/nrp.2014.8.3.284

[30] Lee, H., Choi, J., Shin, S.S. and Yoon, M. (2016) Effects of Korean Red Ginseng ( Panax ginseng) on Obesity and Adipose Inflammation in Ovariectomized Mice. Journal of Ethnopharmacology, 178, 229-237.

[31] Park, J.S., Shin, K.A., Jung, J.S., Hyun, J.W., Van Le, T.K., Kim, D.H., Park, E.M. and Kim, H.S. (2012) Anti-Inflammatory Mechanism of Compound K in Activated Microglia and Its Neuroprotective Effect on Experimental Stroke in Mice. Journal of Pharmacology and Experimental Therapeutics, 341, 59-67.

https://doi.org/10.1124/jpet.111.189035 
[32] Biegert, C., Wagner, I., Lüdtke, R., Kötter, I., Lohmüller, C., Günaydin, I., Taxis, K. and Heide, L. (2004) Efficacy and Safety of Willow Bark Extract in the Treatment of Osteoasrthritis and Rheumatoid Arthritis: Results of 2 Randomized Double-Blind Controlled Trials. The Journal of Rheumatology, 31, 2121-2130.

[33] Khayyal, M.T., El-Ghazaly, M.A., Abdallah, D.M., Okpanyi, S.N., Kelber, O. and Weiser, D. (2005) Mechanisms Involved in the Anti-Inflammatory Effect of a Standardized Willow Bark Extract. Arzneimittelforschung, 55, 677-687.

Submit or recommend next manuscript to SCIRP and we will provide best service for you:

Accepting pre-submission inquiries through Email, Facebook, LinkedIn, Twitter, etc. A wide selection of journals (inclusive of 9 subjects, more than 200 journals) Providing 24-hour high-quality service User-friendly online submission system Fair and swift peer-review system Efficient typesetting and proofreading procedure Display of the result of downloads and visits, as well as the number of cited articles Maximum dissemination of your research work

Submit your manuscript at: http://papersubmission.scirp.org/

Or contact ajps@scirp.org 